\title{
A comparison of recognition memory to numerical decision: How prior probabilities affect cutoff location
}

\author{
ALICE F. HEALY \\ Yale University, New Haven, Connecticut 06520 and \\ Haskins Laboratories, New Haven, Connecticut 06511 \\ and \\ MICHAEL KUBOVY \\ Yale University, New Haven, Connecticut 06520
}

\begin{abstract}
In two experiments, a recognition memory task, in which subjects judged whether a given five-digit number had been shown previously, was compared to a numerical decision task, in which subjects judged whether a given five-digit number represented the height of a man or a woman. Subjects were found to shift $\beta$ (the cutoff point along the decision axis) with changes in prior probabilities in the direction specified by signal detection theory in the numerical decision task but not in the recognition memory task. An explanation of the results, in terms of a difference in d' (discriminability) between the two tasks, was ruled out. In contrast, explanations which cannot be ruled out involve differences between the tasks in the amount of practice required for the subject to learn the manner in which the stimuli are distributed along the decision continuum, and differences in the availability of an alternative basis for response other than the decision continuum.
\end{abstract}

According to the theory of signal detection as it has been applied to recognition memory, subjects who are asked to decide whether a given test item is old should consider the prior probability that the test item is old. Specifically, subjects should raise the cutoff point $(\beta)$ along the decision continuum if the prior probability of an old item decreases, thereby lowering the frequency of the response "old." However, Murdock (1974) reviews an unpublished study by Donaldson where, contrary to the specifications of signal detection theory, different prior probabilities did not lead to different cutoff points in a continuous recognition procedure. Murdock also describes an unpublished recognition memory study by Wells, in which prior probability did affect $\beta$, but he points out that the changes in prior probability in the study were confounded with other changes in the experimental situation. A more recent study by Healy and Jones (1975) extends the generality of Donaldson's result. In a recognition memory task

This research was supported in part by PHS Grants MH26573 and RR07015 to Yale University and NICHD Grant HD01994 to Haskins Laboratories. We are deeply indebted to Randy Smith, who was responsible for the computer programming of Experiments 1 and 2 and who helped design, conduct, and analyze Experiment 1; Donna Sutliff, who helped design Experiment 1; Deborah Letai, who helped conduct and analyze Experiment 1; Debra Pate, who helped design, conduct, and analyze Experiment 2; and Wendy Brown, who assisted us at numerous phases of this research. We also thank W. K. Estes for his illuminating comments on an earlier version of this paper. Request reprints from Alice F. Healy, Department of Psychology, Yale University, New Haven, Connecticut 06520. with blocked study and test trials, they found that, across changes in prior probabilities, subjects maintained a constant hit rate to false alarm rate ratio (HR/FAR), which varies monotonically with $\beta$. Specifically, in the Healy and Jones experiment, subjects gave a rating from 1 to 4 indicating the extent to which they were certain that the given test item was old. Healy and Jones report that for two of the three cutoffs separating the four ratings, subjects did not shift HR/FAR across blocks of trials when the prior probability of an old item changed from $1 / 2$ to $1 / 4$, even though the subjects were informed of the prior probabilities at the start of each test block and were given feedback after each response.

In a reanalysis of the data from Experiment 2 of the study by Healy and Jones, the index $\beta$ was computed for each test block, with the assumption of normal distributions equal in variance for old and new items along the decision continuum. (In this computation and in all succeeding computations described in this paper, the standard score for a hit rate or a false alarm rate with the value of either exactly 0.00 or 1.00 was taken to be +2.00 or -2.00 , respectively.) The mean value of $\beta$ for the blocks of trials with prior probability of an old item of $1 / 2$ was not significantly different from that for the blocks of trials with prior probability of $1 / 4$ for two of the cutoffs, although it was significant for the third. [For the strict cutoff, $F(1,21)<1$; for the medium cutoff, $F(1,21)=2.24,1 / 2 p=.08$; for the lax cutoff, $F(1,21)=4.07,1 / 2 \mathrm{p}=.03$. Here and in all succeeding cases in this paper where a unidirectional hypothesis was being tested, a one-tailed test was 
employed and is indicated by the notation $1 / 2 \mathrm{p}$.] In contrast, subjects did seem to shift the cutoff as a function of relative block position. The main effect of block position was significant for two of the three cutoffs [for the strict cutoff, $\mathrm{F}(3,63)=4.01, \mathrm{p}=.01$; for the medium cutoff, $F(3,63)=7.86, p=.0002$; for the lax cutoff, $F(3,63)=1.29, p=.3]$. The interaction of the factors of prior probability and block position was not significant for any of the cutoffs. Table 1 presents the mean values of $\beta$ averaged across subjects from all three instruction conditions of the Healy and Jones experiment for each of the three cutoff levels, both as a function of relative block position and as a function of pitior probability level.

The results are especially puzzling given the fact that subjects are clearly able to adopt different cutoff points in this situation, as evidenced by the finding that they differentiated the four rating levels as instructed. The posterior probabilities of an item's being an old item. given a particular rating, were computed by Healy and Jones and found to be significantly different for the four rating levels. Subjects are, therefore, able to hold different cutoffs for different ratings, but nevertheless they do not change the cutoffs with changes in prior probabilities.

As Healy and Jones (1975) note, the results discussed above differ markedly from signal detection results, such as those reported by Swets, Tanner, and Birdsall (1961). In that study, as prior probabilities changed, subjects shifted $\beta$ in the direction specified by signal detection theory. Healy and Jones discussed several important differences between their study and that of Swets et al. which could account for the discrepancy in results. One difference was the rate of change in prior probability levels: Prior probabilities changed slowly (between sessions) in the study by Swets et al., but more quickly (within sessions) in the study by Healy and Jones. Another difference was in the extent of the subjects' training: Subjects were highly practiced in the study by Swets et al., but not in the study by Healy and Jones. The primary difference between the two studies, however, lies in the paradigm employed: Healy and

Table 1

Mean Values of $\beta$ in Experiment 2 of Healy and Jones (1975) for Three Cutoff Levels Both as a Function of Relative Block Position and as a Function of Prior Probability Level

\begin{tabular}{lccc}
\hline & \multicolumn{3}{c}{ Cutoff Level } \\
\cline { 3 - 4 } & Strict & Medium & Lax \\
\hline Relative Block Position & & & \\
$\quad$ 1st & 1.97 & 1.03 & .88 \\
2nd & 2.45 & 1.25 & .86 \\
3rd & 2.87 & 1.63 & .97 \\
4th & 2.83 & 1.81 & 1.01 \\
Prior Probability Level & & & \\
1/2 & 2.56 & 1.35 & .85 \\
1/4 & 2.49 & 1.52 & 1.01 \\
\hline
\end{tabular}

Jones employed a recognition memory task, whereas Swets et al. used a signal detection task. Indeed it has been argued (Lieblich \& Lieblich, 1969) that the decision task per se is an important variable in determining whether or not subjects can shift the cutoff point on the decision axis.

The aim of the present study was to determine whether in fact the nature of the decision task influences the extent of shift in cutoff as a function of changes in prior probabilities. Specifically, a recognition memory task modeled after that of Healy and Jones (1975) is compared to a numerical decision task, for which subjects have been shown in previous studies to shift the cutoff as a function of changes in payoffs (Kubovy, Rapoport, \& Tversky, 1971). The numerical decision task was chosen since it provides an ideal setting for studying binary decision making under uncertainty, because irrelevant sensory and mnemonic requirements are eliminated (see Kubovy \& Healy, in press). The two tasks are made to correspond as closely as possible in terms of such important variables as rate of change in prior probabilities and practice, as well as such less clearly relevant variables as the actual pool of stimuli employed and the sequence of correct responses. A simple binary decision response, rather than a rating response, is employed in each condition, in order to determine whether the previous results of Healy and Jones could be attributed solely to the use of a rating procedure.

\section{EXPERIMENT 1}

\section{Method}

Subjects. Twenty-four Yale University undergraduates, taking a course in introductory psychology, participated for course credit. (Three additional subjects were tested, but the data could not be analyzed because of computer failures.) There were two conditions with 12 subjects each. The placement of subjects into conditions was determined by time of arrival for testing. Each subject was tested individually for one session, approximately $45 \mathrm{~min}$ long.

Apparatus. A Sugarman T-6 CRT terminal, controlled by a PDP-10 computer, was used for the visual display of the stimuli and the collection of the responses.

Stimuli. Two sets of five-digit numbers were constructed by sampling at random from two overlapping normal distributions, with the constraint that no two numbers were identical. The two distributions had means of 16,300 and 17,970, respectively, and they both had a standard deviation of 1,000 . Two hundred and twenty numbers were selected from the first distribution to form the first set of stimuli, and 140 numbers were selected from the second distribution to form the second set. (More stimuli were needed for the first set than for the second because of the use of a prior probability level equal to $1 / 4$; see below.) The resulting first set of numbers had a mean of 16,371 and a standard deviation of 1,014 , and the resulting second set had a mean of 17,900 and a standard deviation of 1,102 . The same stimuli were used in both conditions of the experiment with the exception that 40 of the stimuli, 20 chosen at random from each distribution, were employed in the recognition memory condition, but not in the numerical decision condition. Considering only the numbers that were employed in the numerical decision 
condition, the first set had a mean of 16,371 and a standard deviation of 980 , and the second set had a mean of 17,855 and a standard deviation of 1,104 .

Design and procedure. Each subject's session included eight blocks of trials, with a rest period after the first four.

Recognition memory condition. Each block of trials included a study phase and a test phase. During the study phase, the subject saw 20 stimuli to be remembered, of which, 12 were chosen at random without replacement from the first stimulus set and 8 from the second set. Then, during the test phase, the subject responded to each of 40 stimuli, indicating ("yes" or "no") whether each stimulus had been shown during the study phase. The 40 test items in each block included: (1) either 20 or 10 (depending on the prior probability level) of the current 20 study items (old items), chosen at random in the case of 10 , and (2) either 20 or 30 distractors (new items), including either 8 stimuli chosen at random without replacement from the second set and 12 from the first, or 11 from the second set and 19 from the first. New sets of old and new items were used for each block so that each old item appeared either once or twice (either only at study, or once at study and once at test), and each new item was shown once during the eight-block session.

The prior probability of an old item was $1 / 2$ for four of each subject's eight blocks and 1/4 for the other four. Specifically, in four of the blocks, the test sequences included 20 old and 20 new items, and, in four of the blocks, the test sequences included 10 old and 30 new items. The subject was informed of the prior probability for a given block at the start of each test phase and was immediately tested on this information (see below).

The presentation order of the stimuli in a given block was random. There were two pseudorandom permutations of the eight blocks of trials, six subjects being exposed to each of the two permutations. The permutations were chosen so that across permutations each prior probability was used for each block position, and no more than two successive blocks had the same prior probability. One permutation of prior probability levels was $1 / 2,1 / 4,1 / 4,1 / 2,1 / 4,1 / 2,1 / 2,1 / 4$; the second permutation was $1 / 4,1 / 2,1 / 2,1 / 4,1 / 2,1 / 4,1 / 4,1 / 2$.

The timing within each trial block was as follows: At the start of each study phase the word "study" was shown on the screen for approximately $3 \mathrm{sec}$. (Timing is approximate because the experiment was conducted under a time-sharing system.) The screen was then blank for approximately $1 \mathrm{sec}$. Next appeared the 20 study items, displayed successively, each for approximately $2 \mathrm{sec}$, followed by a $1-\mathrm{sec}$ blank screen. After the 20 study items and blanks were displayed, the test phase began with the word "test" appearing on the screen for approximately $3 \mathrm{sec}$, followed by a blank screen for 1 sec. Then one of the two following messages appeared for approximately 6 sec: " 40 items, 20 old, 20 new," or " 40 items, 10 old, 30 new." The message was immediately followed by a test to insure that the subject attended to the information. The screen was cleared and the following query was displayed: "How many old items, 10 or 20?" The query remained on the screen while the subject entered his or her response on the keyboard. If the answer was incorrect, the screen was cleared and the query was displayed again, and the subject responded again. The process was repeated until the subject made the correct response. Immediately after the subject made the correct response, the following query appeared on the next line of the screen: "How many new items, 20 or 30 ?" The query remained on the screen while the subject responded. Again, the process was repeated until the subject made the correct response. After the subject responded correctly to the second query, the screen was cleared and left blank for approximately $1 \mathrm{sec}$, and then the 40-item test sequence began. Each test item was presented, with the following message below it: "Type answer, $y=y e s, n=$ no." As soon as the subject typed one of the two possible responses for the given test item, the screen was cleared and immediate feedback was provided in the following form: "Correct answer was: yes" or "Correct answer was: no." The feedback appeared for approximately $1 \mathrm{sec}$, after which the screen was cleared and the next test item appeared immediately. After the 40th test item and feedback, the second block started immediately. After the completion of the first four blocks, the message "Rest for 2 minutes" appeared on the screen for approximately $2 \mathrm{~min}$, after which time the fifth block was started. After all eight blocks were completed, the following final message appeared on the screen: "Experiment over. Call experimenter."

Numerical decision condition. The design and procedure of the numerical decision condition were identical to those of the recognition memory condition with the following six exceptions: (1) Each block consisted of a test phase only; no study phase was included. (2) The subject was told that each stimulus represented the height of a person in tenths of millimeters. (3) The subject was to respond to each test item by deciding whether it represented the height of a man (i.e., was drawn from the second set) ("yes") or represented the height of a woman (i.e., was drawn from the first set) ("no"). (4) In fact, the $\mathbf{4 0}$ test items in each block included either 20 or 30 (depending on the prior probability level) stimuli chosen at random without replacement from the first stimulus set (women) and either 20 or 10 stimuli chosen at random without replacement from the second set (men). The presentation order of the stimuli was random, except for the constraint that the sequence of correct responses in the test trials corresponded exactly to the sequence of correct responses in the corresponding trials of the recognition memory condition. (5) The message shown at the start of each test phase was changed to the following: "40 items, 20 men, 20 women" or "40 items, 10 men, 30 women." Also, the queries following the messages were changed to the following: "How many men's heights, 10 or 20?" and "How many women's heights, 20 or 30 ?" (6) At the start of the experiment, the subjects were instructed that "The population of men and women from which these heights are taken is an artificial one, and does not necessarily correspond to any population you know of in the real world. However, it is the case that, as in the real world, the average height of men is greater than the average height of women, and also that nevertheless, as in the real world, some men are shorter than some women, and some women are taller than some men. That is, there is overlap between the women's and men's heights in this population."

\section{Results and Discussion}

The results from the present experiment are summarized in Table 2 in terms of mean values of $\beta$, computed with the assumption of normal stimulus distributions equal in variance (although the assumption is not totally justified for the numerical decision condition because of sampling error), as a function of prior probability level and condition. The values of $\beta$ are averaged across relative block positions and groups of subjects shown different block permutations, since neither of these factors showed a main effect which was statistically significant, or a significant interaction with the effects of prior probability level or condition. The standard error of the entries of Table 2, as determined

Table 2

Mean Values of $\beta$ and $d^{\prime}$ in Experiment 1 as a Function of Condition and Prior Probsbility Level

\begin{tabular}{lrrrr} 
& \multicolumn{2}{c}{$\beta$} & \multicolumn{2}{c}{$\mathrm{d}^{\prime}$} \\
\cline { 2 - 5 } Condition & $1 / 2$ & $1 / 4$ & $1 / 2$ & $1 / 4$ \\
\hline Recognition Memory & 1.36 & 1.35 & .59 & .96 \\
Numerical Decision & 1.26 & 1.65 & 1.32 & 1.18 \\
\hline
\end{tabular}


by an analysis of variance, was .13 for the values of $\beta$. As predicted, the difference between average values of $\beta$ for the two prior probability levels is small and in the direction opposite to that predicted from signal detection theory for the recognition memory condition, but larger and in the predicted direction for the numerical decision condition. The interaction of prior probability level and condition is not significant $[F(1,20)=2.43$, $1 / 2 p=.07]$, although the results are in the direction predicted. However, separate analyses for each condition revealed, on one hand, no statistically significant effect of prior probability levels for the recognition memory condition $[F(1,10) \ll 1]$, and, on the other hand, an effect of prior probability levels which is statistically significant for the numerical decision condition $[F(1,10)=4.89,1 / 2 p=.03]$. Note that, although the difference in $\beta$ values is significant and in the predicted direction for the numerical decision task, it is not as large as that expected under ideal conditions, in which the observer should hold a $\beta$ of 1 for the prior probability level of $1 / 2$ and a $\beta$ of 3 for the prior probability level of $1 / 4$, assuming a symmetric payoff matrix.

Values of d' were also calculated for each block of trials, with the assumption of normal stimulus distributions equal in variance. Table 2 presents the values of $\mathrm{d}^{\prime}$ averaged across subjects and.relative block positions. The standard error of the entries of Table 2 for $d^{\prime}$, as determined by an analysis of variance, was .07 . The values of $\mathrm{d}^{\prime}$ are greater for the numerical decision task (1.25) than for the recognition memory task (.77) $[F(1,20)=11.81, p=.003]$. The difference indicates that the numerical decision task employed here is an easier task, in terms of the discriminability of the two stimulus distributions, than the recognition memory task. The difference in discriminability may be critical, since it is conceivable that subjects may be better able to shift $\beta$ when discriminability is better.

In addition to the difference in $\mathrm{d}^{\prime}$ between conditions, there is also a significant interaction of condition and prior probability level $[\mathrm{F}(1,20)=12.12, \mathrm{p}=.002]$. Specifically, when the prior probability of an old item decreases, $\mathrm{d}^{\prime}$ increases for the recognition memory condition, but decreases for the numerical decision condition. The values of $\mathrm{d}^{\prime}$ are not expected to change with changes in prior probability levels, but such changes might occur if the assumption of equal-variance stimulus distributions were violated, as may be the case for recognition memory, and is certainly the case, although to a small degree due to sampling error, for numerical decision. Note, however, the change in $\mathrm{d}^{\prime}$ with prior probability levels (decrease with lower prior probability levels) is in the opposite direction to that expected (increase with lower prior probability levels) in the numerical decision task if the larger variance of the second stimulus distribution is the sole cause (see Green \& Swets, 1974).

The present results support the notion that the nature of the decision task (recognition memory vs. numerical decision) may determine whether subjects shift the location of the cutoff with changes in prior probability levels. However, a possible confounding factor has intruded, namely, discriminability. The two tasks employed here differed in discriminability: The value of $d^{\prime}$ was significantly lower for the recognition memory condition than for the numerical decision condition. The next experiment explores the relationship between discriminability and the extent of shifts in $\beta$ by systematically varying the discriminability in the numerical decision condition. In particular, two numerical decision conditions are presented, with one (high numerical decision condition) designed to have a discriminability which would be greater $\left(\mathrm{d}^{\prime}=1.5\right.$ for the ideal observer $)$ than that found for recognition memory in Experiment 1, and one (low numerical decision condition) designed to have a discriminability which would be less $\left(\mathrm{d}^{\prime}=.5\right.$ for the ideal observer) than that found for recognition memory. In addition, the distributions are more carefully constructed so that the assumption of equal-variance stimulus distributions is entirely justified, at least for the numerical decision conditions.

\section{EXPERIMENT 2}

\section{Method}

Subjects. Thirty-six members of the Yale University community participated as subjects, thirty of whom were students in a course in introductory psychology, and participated for course credit, and six of whom were paid $\$ 2$ for their participation. Two additional subjects were tested, but the data were not analyzed because of computer failure in one case and performance below the level of chance in the other case (tested in the high numerical decision condition). There were three conditions with 12 subjects each. (Each condition contained two paid subjects.) The placement of subjects into conditions was determined by time of arrival for testing. Each subject was tested individually for one session, approximately $45 \mathrm{~min}$ long.

Stimuli. Three sets of 200 five-digit numbers were constructed to approximate three overlapping normal distributions with equal variance. No two numbers in the first and second or first and third sets were identical. The first set was constructed by choosing the midpoint of each $.5 \%$ band of a normal distribution with a mean of 16,300 and a standard deviation of 1,670. The second set was constructed by adding 835 (.5 the standard deviation) to each five-digit number in the first set, and the third set was constructed by adding 2,505 (1.5 the standard deviation) to each five-digit number in the first set. When a five-digit number would have appeared in both the first and second or both the first and third sets by this procedure, the number was rounded down for the first set and rounded up for the second or third set. Only stimuli from the first and second sets were employed for both the low numerical decision condition and the recognition memory condition, and only stimuli from the first and third sets were employed for the high numerical decision condition.

Each of the four blocks of test trials in the low numerical decision condition with prior probability $1 / 2$ was composed of two stimuli chosen at random without replacement from each $10 \%$ band of the first set and two stimuli chosen at random without replacement from each $10 \%$ band of the second set. Analogously, each of the four blocks of test trials with prior probability $1 / 4$ was composed of three stimuli chosen at random 
without replacement from each $10 \%$ band of the first set and one stimulus chosen at random without replacement from each $10 \%$ band of the second set. The constraints insured maximal uniformity of stimuli across blocks. The order of the 40 stimuli in each block was random.

The same stimuli and presentation orders of stimuli were employed for the high numerical decision condition as were employed for the low numerical decision condition, except that every stimulus from the second set was replaced by the corresponding stimulus from the third set. The stimuli that occurred in a given block of the low numerical decision condition also occurred in the corresponding block of the recognition memory condition, and in addition, one stimulus from each $10 \%$ band of the second set, chosen at random without replacement, was included in each of the four blocks of the recognition memory condition with prior probability $1 / 4$. (Note that four stimuli from each $10 \%$ band of the second set and eight stimuli from each $10 \%$ band of the third set were never employed in the experiment.) Twenty of the stimuli assigned to a given block in the recognition memory condition were randomly chosen to appear in the study phase (and hence to be old items), and either 10 or all 20 of these (depending on the prior probability) were randomly chosen to appear again in the test phase along with the remaining stimuli (new items) chosen for that block. The stimulus presentation order was random except for the constraint that the sequence of correct responses in the test trials of the recognition memory condition corresponded exactly to that employed in the numerical decision conditions. The constraints insured maximal similarity of stimuli across the three experimental conditions.

Apparatus, design, and procedure. The apparatus, experimental design, instructions to subjects, and procedure employed in the present experiment were the same as those employed in Experiment 1 .

\section{Results and Discussion}

A preliminary question of interest is whether the discriminability of the two stimulus distributions in the recognition memory condition was intermediate between those of the two numerical decision conditions of the present experiment. Values of $\mathrm{d}^{\prime}$ were computed for each block of each subject's session and are summarized in Table 3, which shows the mean values of $\mathrm{d}^{\prime}$ as a function of condition and prior probability level. The values shown in Table 3 are averaged across relative block positions and groups of subjects shown different block permutations, since neither of the factors was significant or interacted significantly with the factors of prior probability level or condition. The standard error of the entries of Table 3, as determined by an analysis of variance, was .056. As was anticipated, the average value of $d^{\prime}$ for the recognition memory condition (.72) was intermediate between that for the low numerical decision condition (.41) and that for
Table 3

Mean Values of $d^{\prime}$ in Experiment 2 as a Function of Condition and Prior Probability Level

\begin{tabular}{lcc} 
& \multicolumn{2}{c}{ Prior Probability Level } \\
\cline { 2 - 3 } Condition & $1 / 2$ & $1 / 4$ \\
\hline Recognition Memory & .76 & .68 \\
Low Numerical Decision & .40 & .42 \\
High Numerical Decision & 1.42 & 1.18 \\
\hline
\end{tabular}

the high numerical decision condition (1.30), which, incidentally, were both less than they would be for the ideal observer (.5 and 1.5, respectively). The main effect of condition was significant $[F(2,30)=32.84,1 / 2 p \cong 0]$.

In addition, unexpectedly, there was a significant main effect on $\mathrm{d}^{\prime}$ of prior probability levels $[F(1,30)=4.69, p=.04]$. For both the recognition memory and high numerical decision (but not the low numerical decision) conditions, $d^{\prime}$ decreased with decreases in prior probability level. There was not, however, a significant interaction of condition and prior probability level $[F(2,30)=2.74, p=.08]$. Recall that in Experiment 1 a similar decrease in $d^{\prime}$ was found for the numerical decision condition, but an increase was found for the recognition memory condition. A violation of the assumption that the stimulus distributions are equal in variance cannot account for the present decrease, at least in the high numerical decision condition, since for every test block the stimulus distributions were carefully constructed to be equal in variance. A more likely explanation for the observed decrease in $\mathrm{d}^{\prime}$ with decreases in prior probability is that subjects are less certain where to place the cutoff when prior probabilities for the two distributions are unequal. Such an increase in cutoff variability could lead to an apparent decrease in $\mathrm{d}^{\prime}$. It is difficult, however, to explain the reason that the changes in $\mathrm{d}^{\prime}$ as a function of prior probabilities are in opposite directions for the recognition memory conditions of the two experiments.

Despite the uncertainty about the interpretation of the effect of prior probability level on $\mathrm{d}^{\prime}$, it is of interest to consider the effect of prior probability on $\beta$. The mean values of $\beta$ for the present experiment are summarized in Table 4 as a function of condition, prior probability level, and relative block position. The values shown in Table 4 are averaged across groups of subjects shown different block permutations, since neither this factor nor its interactions with other factors proved to

Table 4

Mean Values of $\beta$ in Experiment 2 as a Function of Condition, Relative Block Position, and Prior Probability Level

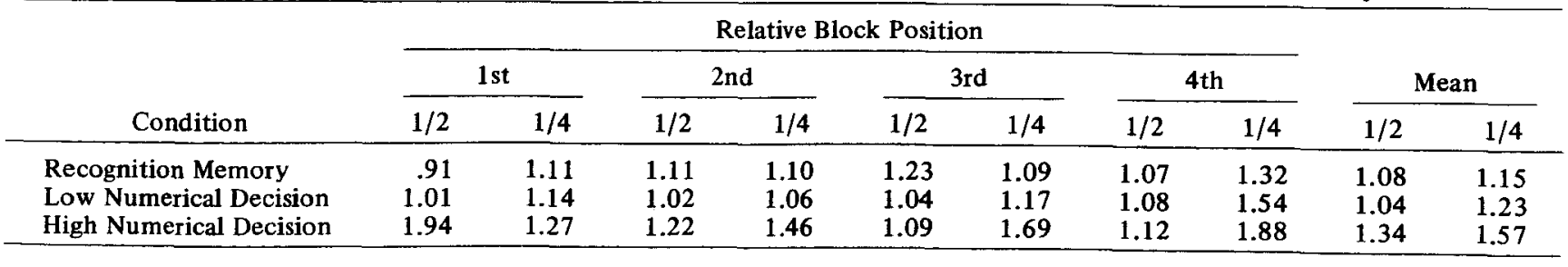


be significant. The standard error of the entries of Table 4 broken down by relative block position, as determined by an analysis of variance, was .13, and the standard error of the entries averaged across block positions was .07 . As predicted, the effect of prior probability level is smaller for the recognition memory condition than for either of the two numerical decision conditions. The effect is in the normatively prescribed direction in each case, but even in the numerical decision conditions, the effect is much smaller than that prescribed for the ideal observer. Although the interaction of condition and prior probability level is not significant $[F(2,30)<1]$, separate analyses of variance revealed a significant effect of prior probability level for the low numerical decision condition $[F(1,10)=19.61$, $1 / 2 \mathrm{p}=.0006]$, but a nonsignificant effect for the high numerical decision condition $[F(1,10)=2.61,1 / 2 p=.07]$ and for the recognition memory condition $[F(1,10)<1]$. In addition, the overall analysis revealed a significant three-way interaction among the factors of condition, prior probability level, and relative block position $[F(6,90)=4.97, p=.0002]$. Specifically, it seems that with changes in prior probability levels, subjects shift $\beta$ in the prescribed direction immediately in the low numerical decision condition, only after some practice (about two blocks long) in the high numerical decision condition, and only after considerable practice (about six blocks long) in the recognition memory condition. The effect of practice suggests that the observed difference in effects of prior probability levels on $\beta$ for different tasks may be eliminated if subjects become well practiced on the tasks.

It is further interesting to note that the effect of prior probability level on $\beta$ in the recognition memory condition is more similar to that in the high numerical decision condition than to that in the low numerical decision condition. The decrease in the effect of prior probability level on $\beta$ cannot, therefore, be attributed to a decrease in discriminability.

\section{SUMMARY AND CONCLUSIONS}

According to signal detection theory, subjects should shift the cutoff point $\beta$ on the decision continuum with changes in prior probabilities. However, it has been found that subjects do not shift $\beta$ in recognition memory tasks with changes in prior probabilities, although they do shift $\beta$ in other situations. The present study sought to determine whether particular task variables, such as rate of change in prior probabilities or amount of practice, were responsible for the result, or, rather, whether the nature of the decision task itself was critical. The recognition memory task was compared to a numerical decision task which provided an ideal setting for decision making since irrelevant mnemonic and sensory factors were eliminated. The two tasks were made as closely analogous as possible in terms of such variables as rate of change in prior probabilities and practice. Nevertheless, subjects were found to shift $\beta$ with changes in prior probabilities in the numerical decision task, but not in the recognition memory task, although in both situations the shifts were much smaller than those prescribed. (Note that conservatism of this type has also been found in signal detection tasks; see, for example, Green \& Swets, 1974.) The possibility that the failure to shift $\beta$ in the recognition memory condition was due to poor discriminability of the stimulus distributions in that situation was ruled out on the basis of finding more consistent shifts in the numerical decision condition with poorer levels of discriminability. Exactly what causes subjects to ignore information about prior probability levels (even when they are forced to attend to such information when it is made available to them) in recognition memory tasks is yet to be understood.

One variable which may be critical is the amount of practice required for the subject to learn the manner in which the stimuli are distributed along the decision continuum. Perhaps more practice is needed for learning the distributions in recognition memory than in numerical decision. The interaction observed in Experiment 2 between condition, prior probability level, and relative block position suggests that, in particular, more practice is needed in recognition memory than in numerical decision for the subject to make adequate use of information about prior probabilities. The possibility that more practice is required for the subjects to learn the distributions in the recognition memory task than in the numerical decision task is surprising, since, presumably, subjects have had more experience outside the laboratory with the decision continuum of familiarity than with that of heights in tenths of millimeters. On the other hand, the subject's assessment of the point at which a given stimulus falls on the familiarity continuum presumably cannot be made as precisely as the assessment of the point at which it falls on the height continuum, and, therefore, more learning might be required in order to reflect prior probabilities.

Another variable which may be critical is the availability of an alternative basis for response other than the decision continuum. It is conceivable that subjects in the recognition memory situation do not in all cases use as a basis for their response for a particular stimulus its value on the familiarity continuum. In some cases, for example, they may be able to rely on a memory scan (for a similar argument see Atkinson \& Juola, 1974). Information about prior probabilities may not be employed when such an alternative response strategy is used. In contrast, it is unlikely that such an alternative response strategy is available to subjects in the numerical decision task.

Other investigators using experimental paradigms very different from those in the present study have also reported that subjects ignore prior probabilities (base rates) in some situations (Kahneman \& Trersky, 
1973). Perhaps the present recognition memory task shares some critical variables with the other situations. It should be noted, however, that the availability of individuating information about each stimulus cannot be the critical variable (as implied by the work of Kahneman \& Tversky, 1973), since such information is availatle to subjects in the numerical decision task as well as in the recognition memory task. Further comparisons of various decision tasks are necessary in order to determine decisively the critical variables influencing the extent to which subjects will be influenced by prior probabilities.

\section{REFERENCES}

Atrinson, R. C., \& Juola, J. F. Search and decision processes in recognition memory. In D. H. Krantz, R. C. Atkinson, R. D. Luce, \& P. Suppes (Eds.), Contemporary developments in mathematical psychology (Vol. I), Leaming, memory, and thinking. San Francisco: W. H. Freeman, 1974.

GreEn, D. M., \& SWETs, J. A. Signal detection theory and psychophysics (reprinted with corrections). Huntington, N.Y: Krieger, 1974.
Healy, A. F., \& Jones, C. Can subjects maintain a constant criterion in a memory task? Memory \& Cognition, 1975, 3, 233-238.

Kahneman, D., \& TVersky, A. On the psychology of prediction. Psychological Review, 1973, 80, 237-251.

Kubovy, M., \& Healy, A. F. The decision rule in probabilistic categorization: What it is and how it is learned. Journal of Experimental Psychology: General, in press.

Kunovy, M., Rapoport, A., \& TVERsKy, A. Deterministic vs. probabilistic strategies in detection. Perception \& Psychophysics, 1971, 9, 427-429.

Lieblich, I., \& Lienlich, A. Effects of different payoff matrices on arithmetical estimation tasks: An attempt to produce "rationality." Perceptual and Motor Skills, 1969, 29, 467-473.

MuRDock, B. B., JR. Human memory: Theory and data. Potomac, Md: Erlbaum, 1974.

Swets, J. A., Tanner, W. P., JR., \& Birdsall, T. G. Decision processes in perception. Psychological Review, $1961,68,301-340$.

(Received for publication June 1, 1976; revision received July 13,1976 .) 